Article

\title{
Lactobacillus plantarum HAC01 Supplementation Improves Glycemic Control in Prediabetic Subjects: A Randomized, Double-Blind, Placebo-Controlled Trial
}

\author{
Mi-Ra Oh ${ }^{1}$, Hui-Yeon Jang ${ }^{1}$, Si-Yeon Lee ${ }^{1}$, Su-Jin Jung ${ }^{1}{ }^{\mathbb{D}}$, Soo-Wan Chae ${ }^{1,2}$, Seung-Ok Lee ${ }^{3, *}$ \\ and Byung-Hyun Park ${ }^{4, * \mathbb{D}}$
}

1 Clinical Trial Center for Functional Foods, Chonbuk National University Hospital, Jeonju 54907, Korea; mroh@jbctc.org (M.-R.O.); janghy@jbctc.org (H.-Y.J.); sylee@jbctc.org (S.-Y.L.); sjjeong@jbctc.org (S.-J.J.); swchae@jbctc.org (S.-W.C.)

2 Biomedical Research Institute, Chonbuk National University Hospital, Jeonju 54907, Korea

3 Division of Gastroenterology and Hepatology, Department of Internal Medicine, Chonbuk National University Medical School, Jeonju 54896, Korea

4 Department of Biochemistry and Molecular Biology, Chonbuk National University Medical School, Jeonju 54896, Korea

* Correspondence: solee@jbnu.ac.kr (S.-O.L.); bhpark@jbnu.ac.kr (B.-H.P.); Tel.: +82-63-250-1289 (S.-O.L.); +82-63-270-3139 (B.-H.P.)

check for updates

Citation: Oh, M.-R.; Jang, H.-Y.; Lee, S.-Y.; Jung, S.-J.; Chae, S.-W.; Lee, S.-O.; Park, B.-H. Lactobacillus plantarum HAC01 Supplementation Improves Glycemic Control in Prediabetic Subjects: A Randomized, Double-Blind, Placebo-Controlled Trial. Nutrients 2021, 13, 2337. https://doi.org/10.3390/nu13072337

Academic Editor: Michael Conlon

Received: 22 May 2021

Accepted: 7 July 2021

Published: 8 July 2021

Publisher's Note: MDPI stays neutral with regard to jurisdictional claims in published maps and institutional affiliations.

Copyright: (c) 2021 by the authors. Licensee MDPI, Basel, Switzerland. This article is an open access article distributed under the terms and conditions of the Creative Commons Attribution (CC BY) license (https:// creativecommons.org/licenses/by/ $4.0 /)$.

\begin{abstract}
A recent animal study demonstrated that administration of Lactobacillus plantarum HAC01 isolated from Korean kimchi improved glycemic control in type 2 diabetic mice. In the present study, we evaluated Lactobacillus plantarum HAC01's effects on metabolic parameters of prediabetic human subjects. Forty subjects with isolated impaired glucose tolerance were randomly assigned to receive a daily placebo $(n=20)$ or a dose of Lactobacillus plantarum HAC01 $(n=20)$ over eight weeks. The primary endpoint was a change in $2 \mathrm{~h}$ postprandial glucose ( $2 \mathrm{~h}-\mathrm{PPG}$ ) levels and the secondary endpoints were assessment of other glucose metabolism parameters, including $\mathrm{HbA1c}$, gut microbiota composition, and fecal short-chain fatty acids (SCFAs). The group with a diet supplemented with Lactobacillus plantarum HAC01 saw a significant reduction in 2h-PPG and $\mathrm{HbA1c}$ levels compared to the placebo group. Fasting plasma glucose, insulin, HOMA-IR, QUICKI, microbiota composition, and fecal SCFAs, however, were not significantly altered. No serious adverse effects were reported. This is the first clinical trial to show a beneficial effect of single-strain probiotic supplementation administered over eight weeks on $\mathrm{HbA1c}$ levels in prediabetic subjects.
\end{abstract}

Keywords: Lactobacillus plantarum; HbA1c; clinical trial; 2h-PPG; prediabetes

\section{Introduction}

Type 2 diabetes (T2D) has become much more prevalent in the last two decades, particularly in young people [1]. According to the International Diabetes Federation, 463 million people were diabetic in 2019, with this number expected to increase to 578 million by 2030 [2]. With proper management, patients can prevent cardiovascular sequelae, including coronary heart disease, stroke, renal failure, and retinopathy [3]. Type 2 diabetes treatments currently focus on alleviating insulin resistance through lifestyle changes and medication. It is difficult, however, to achieve adequate metabolic control with the current generation of treatments, and many patients ultimately fail to respond satisfactorily [4]. There is an urgent need for alternative strategies that both normalize blood glucose levels and increase the rate of treatment success.

Recent meta-analyses have confirmed that alterations to the composition of gut microbiota contribute to the development of insulin resistance in humans [5]. A diminished presence of Firmicutes and relatively higher levels of Bacteroidetes are found in type 2 diabetic patients, as compared to healthy individuals [6]. Altering the composition of gut 
microbiota through probiotics has attracted a great deal of attention among researchers seeking new means of controlling insulin resistance. Bifidobacterium and Lactobacillus species are the gut microbiota most commonly found in probiotics, and their ability to improve glucose homeostasis has been reported in several clinical trials [7-10]. Park et al. [11] recently isolated Lactobacillus plantarum HAC01 from Korean kimchi and observed its weight-lowering effect in high-fat diet-fed mice. The administration of L. plantarum HAC01 also improved glycemic control by restoring gut microbiota composition in diabetic mice, in which T2D was induced by a high-fat diet after the onset of pancreatic dysfunction by streptozotocin administration [12]. These reports indicated that this strain may have the potential to treat T2D in humans. To test this hypothesis, we conducted a randomized, double-blind, placebo-controlled trial in prediabetic subjects. Our primary aim was to investigate the effects of L. plantarum HAC01 on $2 \mathrm{~h}$ postprandial glucose (2h-PPG) levels. Our secondary aims were to investigate its effects on other glucose metabolism-related parameters, gut microbiota composition, and fecal short-chain fatty acids (SCFAs).

\section{Materials and Methods}

\subsection{Ethics}

This study adhered to Consolidated Standards of Reporting Trials (CONSORT) guidelines and was conducted according to the World Medical Association Helsinki Declaration (Version 2013). All subjects gave their written, informed consent before entering the study. The study protocol was approved by the Institutional Review Board of Chonbuk National University Hospital (CUH 2019-03-037) and was registered at the Clinical Research Information Service (CRIS) as No. KCT0005652.

\subsection{Subjects}

A total of 96 participants visited the site, 40 of whom met the inclusion criteria, defined as follows: (1) between 19 and 70 years old; (2) isolated impaired glucose tolerance (I-IGT), which is defined by a 2-h PPG level of between 140 and $199 \mathrm{mg}$ after a $75 \mathrm{~g}$ oral glucose tolerance test (OGTT); and (3) voluntary agreement to participate in the clinical trials and provision of informed consent. Major exclusion criteria were: (1) history of underlying diabetes mellitus; (2) fasting plasma glucose (FPG) levels $\geq 140 \mathrm{mg} / \mathrm{dL}$ or HbA1c $\geq 7.0 \%$; (3) administration of antibiotic within two weeks or corticosteroids in the four weeks prior to the screening visit; (4) administration of hypoglycemic agents, antiobesity drugs, and lipid-lowering agents, etc., in the three months prior to the screening visit; and (5) acute cardiovascular diseases, including myocardial infarction, cardiac insufficiency or stroke, liver failure, acute or chronic renal failure, history of alcohol or drug abuse, pregnancy, or lactation.

\subsection{Study Design}

This randomized, double-blind, placebo-controlled clinical trial was conducted between July 2019 and November 2020 in the Clinical Trial Center for Functional Foods (CTCF2) at Chonbuk National University Hospital. Subjects $(n=40)$ were randomly allocated into L. plantarum HAC01 or placebo groups by a computer-generated random sequence. The intervention was conducted over an eight-week period, during which subjects received either L. plantarum HAC01 or placebo capsules at 4-week intervals, with all subjects instructed to take one capsule per day after their main meal (breakfast, lunch, or dinner). All subjects recorded their daily intake of probiotics or fermented milk products, and subjects were discontinued if they consumed other probiotics or fermented milk products more than four times a week. Subjects were recommended to keep their routine lifestyle, including physical activity and dietary pattern during the eight-week intervention period.

Each test capsule contained a $4 \times 10^{9}$ colony-forming unit (CFU) of L. plantarum HAC01. Dosing was based on the results of a previous animal study that showed improved glycemic control and accounted for the acceptable daily intake of Lactobacillus for humans 
without appreciable side effects [12]. The placebo capsule was composed primarily of microcrystalline cellulose; the flavor, color, appearance, and dosage of the two types of capsules were identical.

The study consisted of a screening visit and three additional visits (V1-V3, 42 days apart between each visit). Demographic data, medical history, and concomitant medications were recorded at the screening visit. OGTTs were also performed to confirm that eligibility criteria were satisfied. At Visit 1 (Day 0) and Visit 3 (Day 84), fecal and blood samples were collected. Vital signs, anthropometric data, physical activity, dietary intake, alcohol consumption, and adverse events were assessed at every visit. Fecal samples were obtained either a day prior to the visit or on the morning of the visit; in the former case, the subjects stored their fecal sample in a conventional freezer until the following morning. All subjects submitted approximately $5 \mathrm{~g}$ of fecal samples in a dedicated stool collection kit containing an ice pack. Fecal samples were stored at $-70^{\circ} \mathrm{C}$ and extracted by the addition of $1 \mathrm{~mL}$ of ice-cold $100 \%$ methanol. Each sample was homogenized (frequency $=30 \mathrm{~Hz}$ ) for $10 \mathrm{~min}$ with a Retsch MM400 mixer mill (Retsch $\mathrm{GmbH}$, Haan, Germany), after which each sample was centrifuged for $10 \mathrm{~min}$ at $4{ }^{\circ} \mathrm{C}$ and $17,000 \mathrm{rpm}$. The supernatants were passed through a $0.2 \mu \mathrm{m}$ PTFE filter and transferred to Eppendorf tubes, then completely dried with a speed vacuum machine.

\subsection{Biochemical Measurements}

OGTTs were administered using a standardized protocol at the screening visit and Visit 3 (Day 84). Briefly, after an overnight fast, subjects consumed a 75 g glucose solution, and blood samples were collected from the median cubital vein and placed in sodium fluoride tubes at $0,30,60,90$, and $120 \mathrm{~min}$ after glucose loading. The $0 \mathrm{~min}$ blood sample was used to determine FPG, insulin levels, HbA1c, triglyceride (TG), total cholesterol (TC), HDL-cholesterol (HDL-C), LDL-cholesterol (LDL-C), adiponectin, and leptin. Glucose metabolism-related parameters were evaluated using the plasma glucose absolute maximum concentration $\left(\mathrm{C}_{\max }\right)$ and incremental area under the curve (iAUC). The iAUC was calculated using the trapezoidal rule for plasma glucose for each subject [13]. Plasma glucose levels were measured using the glucose oxidase method. Insulin concentration was measured using a Cobas e601 module (Hitachi High-Technologies, Tokyo, Japan). HbA1c levels were measured using an automated glycosylated hemoglobin analyzer (ARKRAY Factory, Shiga, Japan). Insulin resistance was estimated through a homeostasis model assessment [14]: HOMA-IR $=$ [FPG $(\mathrm{mg} / \mathrm{dL}) \times$ fasting insulin $(\mu \mathrm{U} / \mathrm{mL}) / 405]$. Insulin sensitivity was estimated by a quantitative insulin sensitivity check index (QUICKI) [15]: QUICKI $=1 /[\log ($ fasting insulin $(\mu \mathrm{U} / \mathrm{mL}))+\log (\mathrm{FPG}(\mathrm{mg} / \mathrm{dL}))]$. Lipid profiles were measured with a Hitachi $7600-110^{\circledR}$ analyzer (Hitachi High-Technologies). Adiponectin was measured by enzyme-linked immunosorbent assay (Molecular Devices, San Jose, CA, USA) and leptin levels were quantified by a double-antibody radioimmunoassay method (Molecular Devices).

\subsection{Analysis of Fecal Microbiota}

Fecal samples were obtained from all 40 subjects prior and subsequent to the intervention. Total fecal DNA was extracted using a QIAamp PowerFecal Pro DNA Kit (Qiagen, Hilden, Germany). The V4 hypervariable regions of the bacterial 16S rRNA were amplified with unique 8 bp barcodes and sequenced on the Illumina MiSeq PE300 platform [16]. Raw reads were analyzed using the QIIME pipeline [17]. Sequences were filtered and clustered into operational taxonomic units at 97\% sequence identity according to the SILVA 132 database [18]. The operational taxonomic units were identified at the phylum to genus levels. The weighted UniFrac distances were used for PCoA [19].

\subsection{Analysis of Fecal Short-Chain Fatty Acid}

Feces samples $(1.4 \mathrm{mg})$ were extracted by the addition of $25 \mu \mathrm{L}$ of 3-nitrophenylhydrazine and $25 \mu \mathrm{L}$ of N-3-dimethylaminopropyl- $\mathrm{N}^{\prime}$-ethylcarbodiimide. Each mixture sample was 
homogenized for 30 min with a Retsch MM400 mixer mill (Retsch $\mathrm{GmbH}$ ). After centrifugation at 14,000 rpm for $5 \mathrm{~min}$, LC-Triple Q-MS analysis (analytical concentration $=2.8 \mathrm{mg} / \mathrm{mL}$ ) was performed on a Waters BEH C ${ }_{18}$ UPLC column $(1.7 \mu \mathrm{m}, 2.1 \mathrm{~mm} \times 100 \mathrm{~mm}$, Waters, Milford, MA, USA), using water/formic acid (100:0.01, $v / v$; solvent A) and acetonitrile/formic acid (100:0.01, v/v; solvent $\mathrm{B})$ as the mobile phase for gradient elution.

\subsection{Sample Size and Statistical Analysis}

We determined the necessary sample size pursuant to methods developed in prior similar studies [20,21]. Based on a desired power of $80 \%$, an alpha error probability of 0.05 , and an estimated effect size of $d=1.3$, we determined that 32 subjects were required. Anticipating a potential dropout rate of $20 \%$, we enrolled 40 subjects in this study.

All analysis was performed based on the per-protocol (PP) set approach with the use of the SAS statistical software version 9.4 (SAS Institute, Cary, NC, USA). Categorical variables were summarized by frequency and proportions, and continuous variables were summarized by the mean and standard deviation (SD) or median and interquartile range (IQR). For between-group comparisons, the $\chi^{2}$ or Fisher exact test was used for categorical variables, and the independent samples $t$-test was used for differences between continuous variables as appropriate. A value of $p<0.05$ was considered significant.

\section{Results}

\subsection{Subjects Characteristics}

Forty subjects were randomly assigned into two groups receiving either L. plantarum HAC01 $(n=20)$ or placebo $(n=20)$. After randomization, three subjects were excluded from the analysis set due to withdrawn consent $(n=2)$ and administration of a prohibited drug $(n=1)$. The per-protocol set, therefore, ultimately included a total of 37 subjects (Figure 1). Baseline characteristics were similar between the two groups (as shown in Table 1).

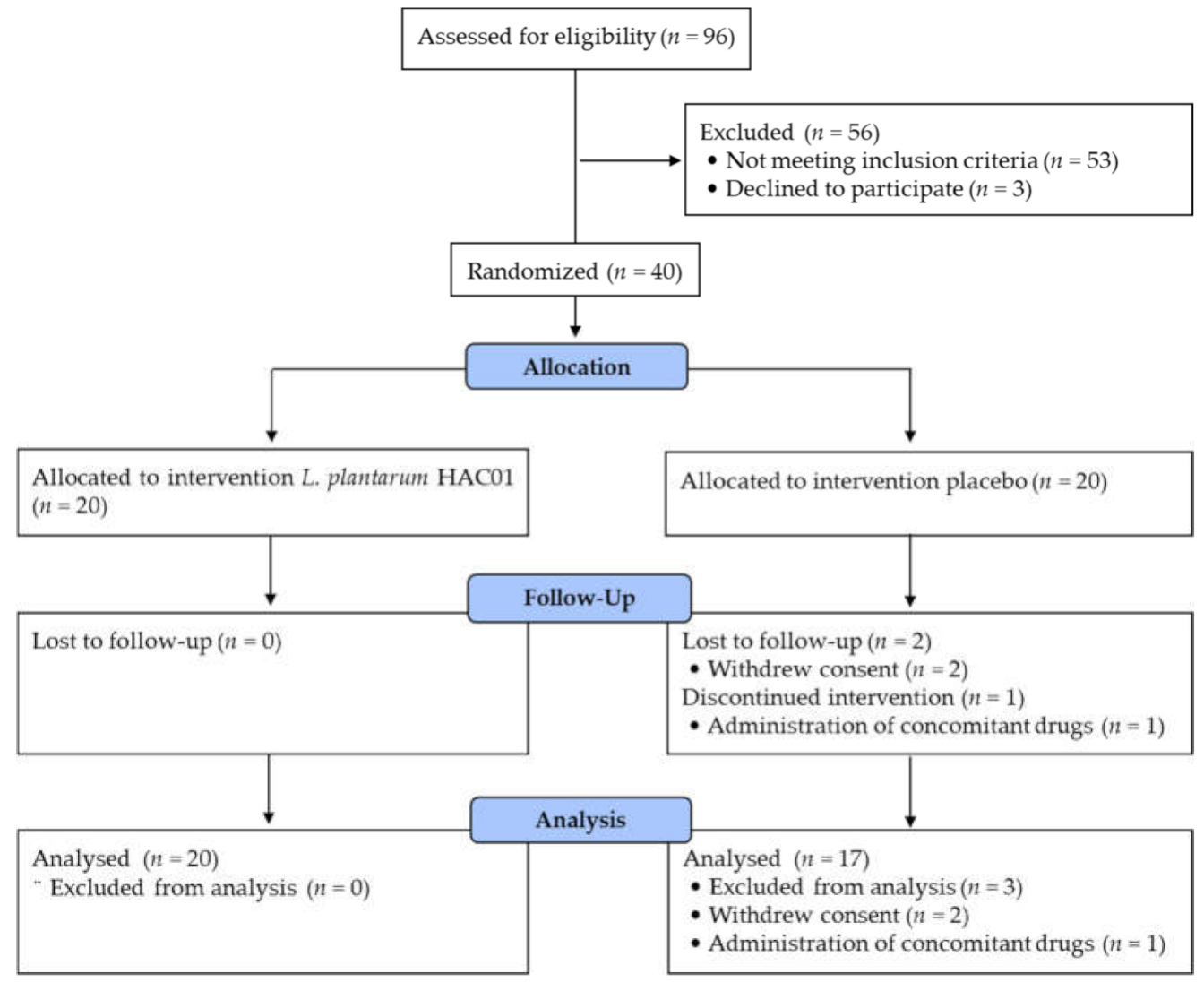

Figure 1. Flow diagram of subject enrollment, allocation, follow-up, and analysis. 
Table 1. Baseline participant clinical characteristics and demographics.

\begin{tabular}{lccc}
\hline & Placebo $(\boldsymbol{n}=\mathbf{2 0})$ & $\begin{array}{c}\text { L. plantarum HAC01 } \\
(\boldsymbol{n}=\mathbf{2 0})\end{array}$ & $\boldsymbol{p}$-Value \\
\hline Age, years & $53.55 \pm 10.18$ & $56.40 \pm 11.57$ & 0.413 \\
Sex, female, $n(\%)$ & $17(85.0)$ & $14(70.0)$ & 0.451 \\
Height, cm & $159.90 \pm 6.07$ & $161.60 \pm 8.74$ & 0.479 \\
Weight, kg & $63.99 \pm 5.68$ & $66.53 \pm 14.09$ & 0.462 \\
BMI, kg/m & $25.03 \pm 1.92$ & $25.25 \pm 3.14$ & 0.793 \\
WHR & & & \\
$\quad$ Female & $0.91 \pm 0.05$ & $0.93 \pm 0.04$ & 0.149 \\
$\quad$ Male & $0.91 \pm 0.03$ & $0.95 \pm 0.02$ & 0.066 \\
Current smoker, $n$ (\%) & $2(10.0)$ & $0(0.0)$ & 0.487 \\
Alcohol consumption, unit/day & $6.81 \pm 5.16$ & $7.32 \pm 12.02$ & 0.922 \\
Physical activity, & 1940 & 980 & 0.762 \\
MET-min/week & $(830-2720)$ & $(440-3060)$ & 0.249 \\
FPG, mg/dL & $101.65 \pm 8.21$ & $99.00 \pm 5.90$ & 0.066 \\
2h-PPG, mg/dL & $161.95 \pm 14.69$ & $172.15 \pm 19.09$ & 0.930 \\
HbA1c, \% & $5.94 \pm 0.38$ & $5.93 \pm 0.33$ & \\
\hline BMI, & & & \\
\hline
\end{tabular}

BMI, body mass index; WHR, waist-hip circumference ratio; MET, metabolic equivalent of task; FPG, fasting plasma glucose; PPG, postprandial plasma glucose. MET is presented as the median (interquartile range).

\subsection{Parameters of Glucose Metabolism}

Tables 2 and 3 and Figure 2 present the change in parameters related to glucose metabolism, including FPG, $2 \mathrm{~h}-\mathrm{PPG}, \mathrm{iAUC}_{0-2 \mathrm{~h}}, \mathrm{HbA1c}$, fasting insulin, HOMA-IR, and QUICKI. Compared to the placebo group, a significant reduction in 2h-PPG and $\mathrm{HbA1c}$ levels was observed in the group administered L. plantarum HAC01 after 8 weeks (Table 1 and Figure 2). There were no significant differences between the L. plantarum HAC01 and placebo groups in FPG, fasting insulin, HOMA-IR, and QUICKI (Tables 2 and 3).



Figure 2. Effect of L. plantarum HAC01 supplementation on the change (\%) of HbA1c levels from the baseline value (adjusted to zero) to Week 8 . 
Table 2. Change in blood glucose levels during the intervention period.

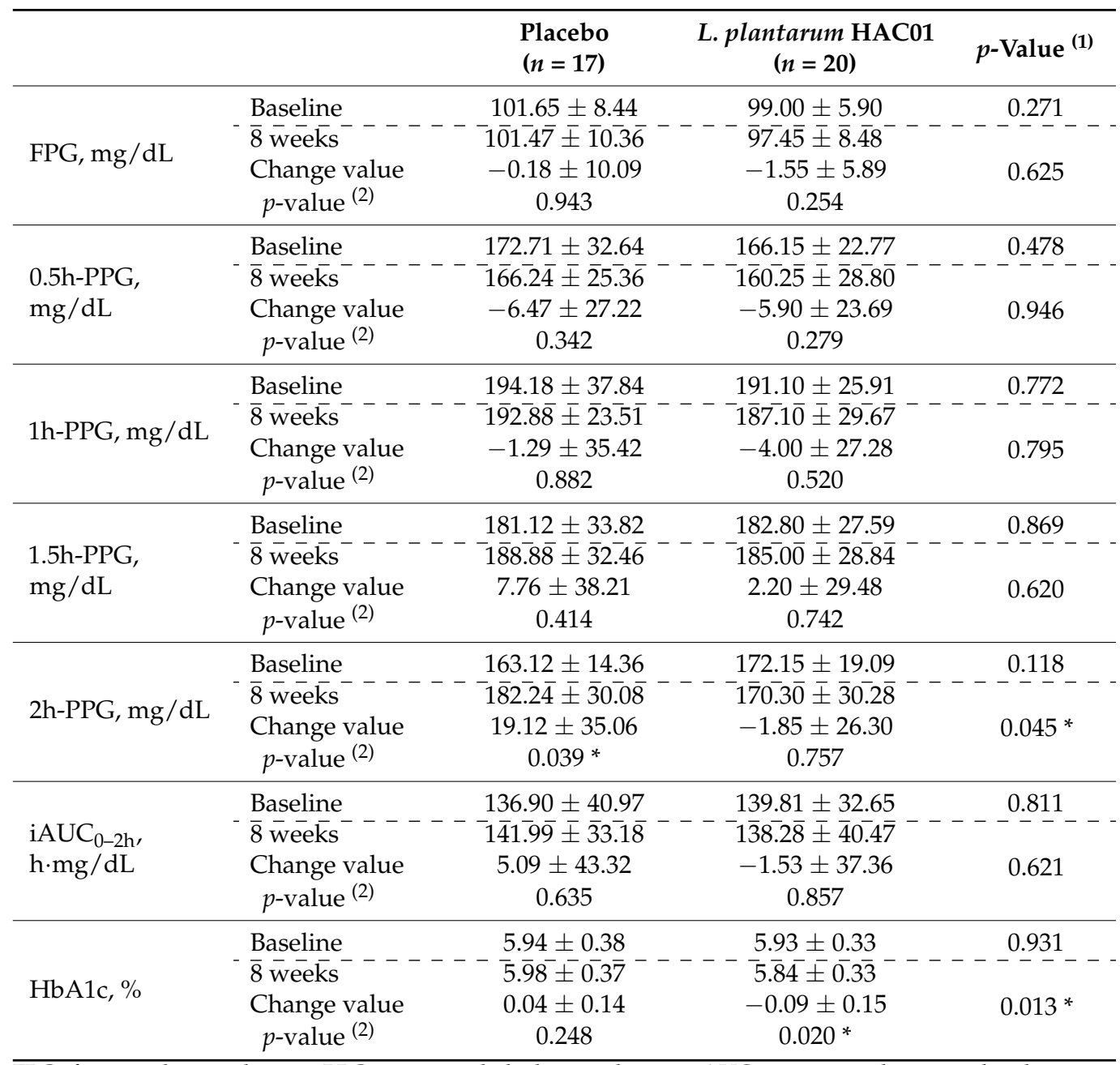

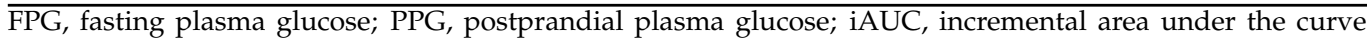
$p$-value $<0.05$ are denoted with an asterisk $\left(^{*}\right) .{ }^{(1)}$ Compared between groups; $p$-value by independent $t$-test. (2) Compared within group; $p$-value by paired $t$-test.

Table 3. Change of insulin, HOMA-IR, and QUICKI during the intervention period.

\begin{tabular}{|c|c|c|c|c|}
\hline & & $\begin{array}{l}\text { Placebo } \\
(n=17)\end{array}$ & $\begin{array}{l}\text { L. plantarum HAC01 } \\
(n=20)\end{array}$ & $p$-Value ${ }^{(1)}$ \\
\hline \multirow{4}{*}{ Insulin, $\mu \mathrm{U} / \mathrm{mL}$} & Baseline & $11.54 \pm 5.29$ & $9.63 \pm 4.24$ & 0.230 \\
\hline & $\overline{8}$ wee $\overline{\text { eks }}--$ & $\overline{1} 1.4 \overline{4} \pm \overline{6} . \overline{17}$ & $\overline{9} . \overline{5} \overline{3} \pm \overline{4.0 \overline{5}}$ & \\
\hline & Change value & $-0.10 \pm 5.34$ & $-0.09 \pm 3.06$ & 0.998 \\
\hline & $p$-value ${ }^{(2)}$ & 0.941 & 0.892 & \\
\hline \multirow{4}{*}{ HOMA-IR } & Baseline & $2.93 \pm 1.42$ & $2.36 \pm 1.06$ & 0.166 \\
\hline & $\overline{8}$ weeks & $\overline{2} . \overline{9} 1 \pm 1.6 \overline{0}$ & $2.35 \pm 1.10$ & \\
\hline & Change value & $-0.02 \pm 1.56$ & $0.00 \pm 0.86$ & 0.958 \\
\hline & $p$-value ${ }^{(2)}$ & 0.949 & 0.992 & \\
\hline \multirow{4}{*}{ QUICKI } & Baseline & $0.33 \pm 0.03$ & $0.34 \pm 0.03$ & 0.268 \\
\hline & $\overline{8}$ weèks ${ }^{-}$ & $\overline{0 . \overline{3}} \overline{4} \pm 0.0 \overline{4}$ & $\overline{0.3 \overline{4} \pm 0.0 \overline{3}}$ & \\
\hline & Change value & $0.01 \pm 0.03$ & $0.00 \pm 0.02$ & 0.761 \\
\hline & $p$-value ${ }^{(2)}$ & 0.515 & 0.621 & \\
\hline
\end{tabular}

HOMA-IR, homeostatic model assessment for insulin resistance; QUICKI, quantitative insulin sensitivity check index. ${ }^{(1)}$ Compared between groups; $p$-value by independent $t$-test. ${ }^{(2)}$ Compared within group; $p$-value by paired $t$-test. 


\subsection{Lipid Profiles, Adiponectin, and Leptin}

After eight weeks of intervention, there were no significant differences in TC, HDL-C, LDL-C, TG, adiponectin, and leptin between the L. plantarum HAC01 and placebo groups (data not shown).

\subsection{Fecal Microbiota Composition and SCFAs}

To determine the effects of L. plantarum HAC01 supplementation on the fecal microbial community, the bacterial composition in the feces was analyzed. Using a weighted UniFrac distance matrix, beta-diversity was examined through PCoA, with the results showing no significant difference between the study groups (data not shown). Consistent with these results, the fecal concentration of total SCFAs, as well as acetate, propionate, iso-butyrate, butyrate, iso-valerate, and valerate did not significantly differ between the study groups (data not shown).

\subsection{Safety}

Seven adverse events occurred during the intervention, none of which were serious. The proportion of subjects reporting an adverse event was similar in each study group (L. plantarum HAC01, $n=3$; placebo, $n=4$ ). Other safety parameters (vital signs, ECG, and laboratory data) in the L. plantarum HAC01 group were not significantly altered over the course of the study period (data not shown).

\section{Discussion}

The American Diabetes Association (ADA) defines prediabetes as existing when one of three distinct conditions are satisfied: impaired fasting glucose (IFG, FPG levels of 100-125 mg/dL), IGT (2h-PPG levels of 140-199 mg/dL during an OGTT), or HbA1c $(5.7-6.4 \%)$ [22]. IFG and IGT can appear in isolation (I-IFG or I-IGT) or in combination (IFG + IGT). The ADA's expert panel estimates that up to $70 \%$ of prediabetic individuals will eventually be diagnosed with T2D [23]. Thus, it is important to encourage prediabetic subjects to keep a healthy lifestyle to prevent or delay the onset of T2D. Both lifestyle and pharmacological interventions may improve this situation if implemented prior to the development of diabetes [24-26]. In this study, we specifically targeted patients with I-IGT, as this category of prediabetes is predominantly associated with unhealthy eating habits and physical inactivity, in contrast with I-IFG, which is more closely associated with male sex and other genetic factors $[27,28]$. Daily administration of L. plantarum HAC01 to subjects with I-IGT over an eight-week period significantly improved 2h-PPG (primary endpoint) and $\mathrm{HbA1c}$ (secondary endpoint) levels as compared to the placebo group. There was, however, no significant difference in FPG levels between the study groups, suggesting that $L$. plantarum HAC01 may be effective in prediabetic subjects with I-IGT, but not those with I-IFG.

Recent clinical trials exploring the use of various formulations of multistrain probiotics over 12-week periods resulted in significant reductions in $\mathrm{HbA1c}$ levels in subjects with T2D $[10,29,30]$. In contrast, two previous clinical trials using single-strain probiotics (Lactobacillus casei or Lactobacillus reuteri) failed to reduce HbA1c levels [9,31]. Taken as a whole, reports suggest that multistrain probiotics are more effective than single-strain probiotics at $\mathrm{HbA} 1 \mathrm{c}$ reduction.

We observed a clear improvement in HbA1c levels after only eight weeks of L. plantarum HAC01 supplementation. As this finding was surprising, and aware that probiotics achieve their metabolic benefits through the modulation of the gut microbiota composition [32], we analyzed the gut microbiota composition. In contrast with the results of our animal study, in which L. plantarum HAC01 increased the presence of the Akkermansiaceae family and decreased the presence of the Desulovibrionaceae family in mice feces [12], we observed no changes in the abundance or taxonomic composition of the human fecal microbiome. These results suggest that it is microbial products, and not a difference in microbiota composition, that perform a regulatory role in glycemic control in humans. However, the 
interpretation of the results related to the microbial composition of the feces must proceed with caution, as fecal microbiota may not accurately reflect intestinal mucosa-associated microbiota, and prolonged storage of stool specimens, even at $-70{ }^{\circ} \mathrm{C}$, may have led to changes in the composition of the samples [33,34].

Recent studies have implicated SCFAs (including acetate, propionate, and butyrate) as primary microbial-derived metabolites that connect gut microbiota with their healthpromoting effects [35]. SCFA binds to the receptors of free fatty acids, such as GPR41 and GPR43, and increases insulin sensitivity and pancreatic $\beta$-cell proliferation [36]. A comparison of baseline and Week 8 values confirmed no changes in the plasma concentrations of various SCFAs in subjects who received L. plantarum HAC01, suggesting that circulating SCFAs derived from gut microbiota were likely not responsible for the observed improvements in $\mathrm{HbA} 1 \mathrm{c}$ levels. However, this conclusion warrants further investigation, as SCFAs have elsewhere been proposed as the most probable mechanism by which probiotics promote health outcomes [35] and fecal levels of SCFAs are affected by factors as diverse as microbiota composition, colonic transit time, luminal $\mathrm{pH}$, host health status, and colonic clearance [37-39]. Moreover, the number of subjects in each group we sampled was small. Accordingly, our results should be treated as preliminary. Larger scale studies will validate these issues and assess the exact mechanism(s) by which L. plantarum HAC01 influences glucose parameters.

In this study, the primary endpoint was a change in 2h-PPG levels and intergroup comparison showed a significant improvement by L. plantarum HAC01 supplementation $(p=0.045)$. However, an intragroup comparison revealed no significant improvement in $2 \mathrm{~h}$ PPG levels after 8 weeks of L. plantarum HAC01 supplementation. Subjects in the placebo group exhibited relatively low baseline levels of $2 \mathrm{~h}$-PPG compared with the L. plantarum HAC01-supplemented group. These results indicate that the observed improvement in $2 \mathrm{~h}$ PPG levels was not a result of L. plantarum HAC01's insulin sensitizing effects. Seemingly confirming this, we observed that plasma insulin levels, HOMA-IR, and QUICKI were also unchanged by the administration of L. plantarum HAC 01 .

This study has some limitations. Though sufficiently powered, the total number of subjects that participated was not large. As the primary aim of this study was to determine whether single-strain probiotic L. plantarum HAC01 was beneficial to prediabetic subjects, the study size was sufficient to achieve this goal. Additionally, we did not measure a doseresponse relationship. Further studies with a larger sample size and multiple doses will be required to determine whether L. plantarum HAC01 is capable of consistently achieving adequate glycemic control in prediabetic humans.

\section{Conclusions}

This clinical study confirmed the results of recent animal studies and demonstrated that an eight-week course of L. plantarum HAC01 supplementation significantly improved $\mathrm{HbA1c}$ and 2h-PPG levels relative to placebo in prediabetic subjects. No serious adverse effects were observed, suggesting that L. plantarum HAC01 has potential as an effective lifestyle intervention to forestall or prevent the onset of T2D.

Author Contributions: Conceptualization, formal analysis, investigation, and writing-original draft preparation, M.-R.O., H.-Y.J., S.-Y.L., and S.-J.J.; funding acquisition and project administration, S.-W.C.; conceptualization, formal analysis, writing—original draft preparation, writing-review and editing, and supervision, S.-O.L. and B.-H.P. All authors have read and agreed to the published version of the manuscript.

Funding: This work was supported by a grant (2016M3A9A592316023) from the National Research Foundation of Korea (NRF) funded by the Korean government (MSIP).

Institutional Review Board Statement: The study was conducted according to the guidelines of the Declaration of Helsinki and approved by the Institutional Review Board of Chonbuk National University Hospital (CUH2019-03-037, 22 April 2019).

Informed Consent Statement: Informed consent was obtained from all subjects involved in the study. 
Data Availability Statement: The datasets generated during and/or analyzed during the current study are available from the corresponding author on reasonable request.

Acknowledgments: The authors would like to thank the Chonbuk National University Writing Center for its skilled proofreading service.

Conflicts of Interest: The authors declare no conflict of interest.

\section{References}

1. Haddad, J.A.; Haddad, A.N. The past decade in type 2 diabetes and future challenges. Hormones 2018, 17, 451-459. [CrossRef]

2. Thomas, R.L.; Halim, S.; Gurudas, S.; Sivaprasad, S.; Owens, D.R. IDF Diabetes Atlas: A review of studies utilising retinal photography on the global prevalence of diabetes related retinopathy between 2015 and 2018. Diabetes Res. Clin. Pract. 2019, 157, 107840. [CrossRef]

3. Chatterjee, S.; Khunti, K.; Davies, M.J. Type 2 diabetes. Lancet 2017, 389, 2239-2251. [CrossRef]

4. Wang, C.Y.; Neil, D.L.; Home, P. 2020 vision-An overview of prospects for diabetes management and prevention in the next decade. Diabetes Res. Clin. Pract. 2018, 143, 101-112. [CrossRef] [PubMed]

5. Kocsis, T.; Molnar, B.; Nemeth, D.; Hegyi, P.; Szakacs, Z.; Balint, A.; Garami, A.; Soos, A.; Marta, K.; Solymar, M. Probiotics have beneficial metabolic effects in patients with type 2 diabetes mellitus: A meta-analysis of randomized clinical trials. Sci. Rep. 2020, 10, 11787. [CrossRef]

6. Larsen, N.; Vogensen, F.K.; van den Berg, F.W.; Nielsen, D.S.; Andreasen, A.S.; Pedersen, B.K.; Al-Soud, W.A.; Sorensen, S.J.; Hansen, L.H.; Jakobsen, M. Gut microbiota in human adults with type 2 diabetes differs from non-diabetic adults. PLoS ONE 2010, 5, e9085. [CrossRef]

7. Toshimitsu, T.; Gotou, A.; Sashihara, T.; Hachimura, S.; Shioya, N.; Suzuki, S.; Asami, Y. Effects of 12-week ingestion of yogurt containing Lactobacillus plantarum OLL2712 on glucose metabolism and chronic inflammation in prediabetic adults: A randomized placebo-controlled trial. Nutrients 2020, 12, 374. [CrossRef] [PubMed]

8. Sabico, S.; Al-Mashharawi, A.; Al-Daghri, N.M.; Wani, K.; Amer, O.E.; Hussain, D.S.; Ahmed Ansari, M.G.; Masoud, M.S.; Alokail, M.S.; McTernan, P.G. Effects of a 6-month multi-strain probiotics supplementation in endotoxemic, inflammatory and cardiometabolic status of T2DM patients: A randomized, double-blind, placebo-controlled trial. Clin. Nutr. 2019, 38, 1561-1569. [CrossRef] [PubMed]

9. Mobini, R.; Tremaroli, V.; Stahlman, M.; Karlsson, F.; Levin, M.; Ljungberg, M.; Sohlin, M.; Berteus Forslund, H.; Perkins, R.; Backhed, F.; et al. Metabolic effects of Lactobacillus reuteri DSM 17938 in people with type 2 diabetes: A randomized controlled trial. Diabetes Obes. Metab. 2017, 19, 579-589. [CrossRef] [PubMed]

10. Madempudi, R.S.; Ahire, J.J.; Neelamraju, J.; Tripathi, A.; Nanal, S. Efficacy of UB0316, a multi-strain probiotic formulation in patients with type 2 diabetes mellitus: A double blind, randomized, placebo controlled study. PLoS ONE 2019, 14, e0225168. [CrossRef]

11. Park, S.; Ji, Y.; Jung, H.Y.; Park, H.; Kang, J.; Choi, S.H.; Shin, H.; Hyun, C.K.; Kim, K.T.; Holzapfel, W.H. Lactobacillus plantarum HAC01 regulates gut microbiota and adipose tissue accumulation in a diet-induced obesity murine model. Appl. Microbiol. Biotechnol. 2017, 101, 1605-1614. [CrossRef]

12. Lee, Y.S.; Lee, D.; Park, G.S.; Ko, S.H.; Park, J.; Lee, Y.K.; Kang, J. Lactobacillus plantarum HAC01 ameliorates type 2 diabetes in high-fat diet and streptozotocin-induced diabetic mice in association with modulating the gut microbiota. Food Funct. 2021, in press. [CrossRef]

13. Wolever, T.M.; Jenkins, D.J.; Jenkins, A.L.; Josse, R.G. The glycemic index: Methodology and clinical implications. Am. J. Clin. Nutr. 1991, 54, 846-854. [CrossRef]

14. Matthews, D.R.; Hosker, J.P.; Rudenski, A.S.; Naylor, B.A.; Treacher, D.F.; Turner, R.C. Homeostasis model assessment: Insulin resistance and beta-cell function from fasting plasma glucose and insulin concentrations in man. Diabetologia 1985, 28, 412-419. [CrossRef]

15. Katz, A.; Nambi, S.S.; Mather, K.; Baron, A.D.; Follmann, D.A.; Sullivan, G.; Quon, M.J. Quantitative insulin sensitivity check index: A simple, accurate method for assessing insulin sensitivity in humans. J. Clin. Endocrinol. Metab. 2000, 85, 2402-2410. [CrossRef] [PubMed]

16. Caporaso, J.G.; Lauber, C.L.; Walters, W.A.; Berg-Lyons, D.; Huntley, J.; Fierer, N.; Owens, S.M.; Betley, J.; Fraser, L.; Bauer, M.; et al. Ultra-high-throughput microbial community analysis on the Illumina HiSeq and MiSeq platforms. ISME J. 2012, 6, 1621-1624. [CrossRef] [PubMed]

17. Caporaso, J.G.; Kuczynski, J.; Stombaugh, J.; Bittinger, K.; Bushman, F.D.; Costello, E.K.; Fierer, N.; Pena, A.G.; Goodrich, J.K.; Gordon, J.I. QIIME allows analysis of high-throughput community sequencing data. Nat. Methods 2010, 7, 335. [CrossRef]

18. Yilmaz, P.; Parfrey, L.W.; Yarza, P.; Gerken, J.; Pruesse, E.; Quast, C.; Schweer, T.; Peplies, J.; Ludwig, W.; Glöckner, F.O. The SILVA and "All-species Living Tree Project (LTP)" taxonomic frameworks. Nucleic Acids Res. 2014, 42, D643-D648. [CrossRef] [PubMed]

19. Lozupone, C.; Lladser, M.E.; Knights, D.; Stombaugh, J.; Knight, R. UniFrac: An effective distance metric for microbial community comparison. ISME J. 2010, 5, 169. [CrossRef] 
20. Asemi, Z.; Alizadeh, S.A.; Ahmad, K.; Goli, M.; Esmaillzadeh, A. Effects of beta-carotene fortified synbiotic food on metabolic control of patients with type 2 diabetes mellitus: A double-blind randomized cross-over controlled clinical trial. Clin. Nutr. 2016, 35, 819-825. [CrossRef]

21. Oh, M.R.; Park, S.H.; Kim, S.Y.; Back, H.I.; Kim, M.G.; Jeon, J.Y.; Ha, K.C.; Na, W.T.; Cha, Y.S.; Park, B.H.; et al. Postprandial glucose-lowering effects of fermented red ginseng in subjects with impaired fasting glucose or type 2 diabetes: A randomized, double-blind, placebo-controlled clinical trial. BMC Complement. Altern Med. 2014, 14, 237. [CrossRef]

22. American Diabetes Association. 2. Classification and diagnosis of diabetes: Standards of medical care in diabetes-2021. Diabetes Care 2021, 44, S15-S33. [CrossRef]

23. Tabak, A.G.; Herder, C.; Rathmann, W.; Brunner, E.J.; Kivimaki, M. Prediabetes: A high-risk state for diabetes development. Lancet 2012, 379, 2279-2290. [CrossRef]

24. Tuomilehto, J.; Lindstrom, J.; Eriksson, J.G.; Valle, T.T.; Hamalainen, H.; Ilanne-Parikka, P.; Keinanen-Kiukaanniemi, S.; Laakso, M.; Louheranta, A.; Rastas, M.; et al. Prevention of type 2 diabetes mellitus by changes in lifestyle among subjects with impaired glucose tolerance. N. Engl. J. Med. 2001, 344, 1343-1350. [CrossRef]

25. Gillies, C.L.; Abrams, K.R.; Lambert, P.C.; Cooper, N.J.; Sutton, A.J.; Hsu, R.T.; Khunti, K. Pharmacological and lifestyle interventions to prevent or delay type 2 diabetes in people with impaired glucose tolerance: Systematic review and meta-analysis. BMJ 2007, 334, 299. [CrossRef]

26. Knowler, W.C.; Barrett-Connor, E.; Fowler, S.E.; Hamman, R.F.; Lachin, J.M.; Walker, E.A.; Nathan, D.M.; Diabetes Prevention Program Research Group. Reduction in the incidence of type 2 diabetes with lifestyle intervention or metformin. N. Engl. J. Med. 2002, 346, 393-403.

27. Oh, M.R.; Jung, S.J.; Bae, E.J.; Park, B.H.; Chae, S.W. Clinical characteristics and associated risk factors of prediabetes in the southwestern region of Korea from 2010-2019. J. Clin. Med. 2020, 9, 1114. [CrossRef] [PubMed]

28. Faerch, K.; Borch-Johnsen, K.; Holst, J.J.; Vaag, A. Pathophysiology and aetiology of impaired fasting glycaemia and impaired glucose tolerance: Does it matter for prevention and treatment of type 2 diabetes? Diabetologia 2009, 52, 1714-1723. [CrossRef] [PubMed]

29. Perraudeau, F.; McMurdie, P.; Bullard, J.; Cheng, A.; Cutcliffe, C.; Deo, A.; Eid, J.; Gines, J.; Iyer, M.; Justice, N.; et al. Improvements to postprandial glucose control in subjects with type 2 diabetes: A multicenter, double blind, randomized placebo-controlled trial of a novel probiotic formulation. BMJ Open Diabetes Res. Care 2020, 8, e001319. [CrossRef]

30. Firouzi, S.; Majid, H.A.; Ismail, A.; Kamaruddin, N.A.; Barakatun-Nisak, M.Y. Effect of multi-strain probiotics (multi-strain microbial cell preparation) on glycemic control and other diabetes-related outcomes in people with type 2 diabetes: A randomized controlled trial. Eur. J. Nutr. 2017, 56, 1535-1550. [CrossRef] [PubMed]

31. Khalili, L.; Alipour, B.; Asghari Jafar-Abadi, M.; Faraji, I.; Hassanalilou, T.; Mesgari Abbasi, M.; Vaghef-Mehrabany, E.; Alizadeh Sani, M. The effects of Lactobacillus casei on glycemic response, serum sirtuin1 and fetuin-A levels in patients with type 2 diabetes mellitus: A randomized controlled trial. Iran. Biomed. J. 2019, 23, 68-77. [CrossRef]

32. Sanchez, B.; Delgado, S.; Blanco-Miguez, A.; Lourenco, A.; Gueimonde, M.; Margolles, A. Probiotics, gut microbiota, and their influence on host health and disease. Mol. Nutr. Food Res. 2017, 61, 1600240. [CrossRef]

33. Ott, S.J.; Musfeldt, M.; Timmis, K.N.; Hampe, J.; Wenderoth, D.F.; Schreiber, S. In vitro alterations of intestinal bacterial microbiota in fecal samples during storage. Diagn. Microbiol. Infect. Dis. 2004, 50, 237-245. [CrossRef] [PubMed]

34. Rochet, V.; Rigottier-Gois, L.; Rabot, S.; Dore, J. Validation of fluorescent in situ hybridization combined with flow cytometry for assessing interindividual variation in the composition of human fecal microflora during long-term storage of samples. J. Microbiol. Methods 2004, 59, 263-270. [CrossRef] [PubMed]

35. Bridgeman, S.C.; Northrop, W.; Melton, P.E.; Ellison, G.C.; Newsholme, P.; Mamotte, C.D.S. Butyrate generated by gut microbiota and its therapeutic role in metabolic syndrome. Pharmacol. Res. 2020, 160, 105174. [CrossRef] [PubMed]

36. Tolhurst, G.; Heffron, H.; Lam, Y.S.; Parker, H.E.; Habib, A.M.; Diakogiannaki, E.; Cameron, J.; Grosse, J.; Reimann, F.; Gribble, F.M. Short-chain fatty acids stimulate glucagon-like peptide-1 secretion via the G-protein-coupled receptor FFAR2. Diabetes 2012, 61, 364-371. [CrossRef] [PubMed]

37. Oufir, L.E.; Barry, J.L.; Flourie, B.; Cherbut, C.; Cloarec, D.; Bornet, F.; Galmiche, J.P. Relationships between transit time in man and in vitro fermentation of dietary fiber by fecal bacteria. Eur. J. Clin. Nutr. 2000, 54, 603-609. [CrossRef]

38. Jorgensen, J.R.; Fitch, M.D.; Mortensen, P.B.; Fleming, S.E. Absorption and metabolism of octanoate by the rat colon in vivo: Concentration dependency and influence of alternative fuels. Gut 2002, 51, 76-81. [CrossRef]

39. Willing, B.P.; Dicksved, J.; Halfvarson, J.; Andersson, A.F.; Lucio, M.; Zheng, Z.; Jarnerot, G.; Tysk, C.; Jansson, J.K.; Engstrand, L. A pyrosequencing study in twins shows that gastrointestinal microbial profiles vary with inflammatory bowel disease phenotypes. Gastroenterology 2010, 139, 1844-1854. [CrossRef] [PubMed] 